\title{
DOS ESPECIES NUEVAS DEL GÉNERO COCCOPHAGUS WESTWOOD, 1833 (HYMENOPTERA: APHELINIDAE) DEL ESTADO DE TAMAULIPAS, MÉXICO
}

\author{
Svetlana Nikolaevna MYARTSEVA, Enrique RUIZ-CANCINO \& JuANa \\ MARÍA CORONADO-BLANCO* \\ Cuerpo Académico Entomología Aplicada, Facultad de Ingeniería y Ciencias, \\ Universidad Autónoma de Tamaulipas, 87149, Cd. Victoria, Tamaulipas, México \\ <smyartse@uat.edu.mx>; <eruiz@uat.edu.mx>; * Autora de correspondencia: <jmcoronado@uat.edu.mx>
}

Myartseva, S. N., Ruiz-Cancino, E. \& Coronado-Blanco, J. M. 2014. Dos especies nuevas del género Coccophagus Westwood, 1833 (Hymenoptera: Aphelinidae) del estado de Tamaulipas, México. Acta Zoológica Mexicana (n.s.), 30(1): 135-143.

RESUMEN. Se describen dos nuevas especies de Aphelinidae del Estado de Tamaulipas, México: Coccophagus indefinitus Myartseva n. sp., un parasitoide de Parasaissetia nigra (Nietner) en el muérdago Phoradendron quadrangulare (Kunth) Griseb., en huizaches Acacia farnesiana (L.) Willd., y en sauces Salix humboldtiana Willd., y de otro Coccidae no identificado; y Coccophagus tamaulipecus Myartseva n. sp., en Coccidae no identificado. Nuevo registro de $P$. nigra en muérdagos y Nuevo registro de Aphelinidae como parasitoides de P. nigra en muérdagos.

Palabras clave: Aphelinidae, Coccophagus, nuevas especies, muérdagos, Tamaulipas, México.

Myartseva, S. N., Ruiz-Cancino, E. \& Coronado-Blanco, J. M. 2014. Two new species of the genus Coccophagus Westwood, 1833 (Hymenoptera: Aphelinidae) from the state of Tamaulipas, Mexico. Acta Zoológica Mexicana (n.s.), 30(1): 135-143.

ABSTRACT. Two new species of Aphelinidae are described from the State of Tamaulipas, Mexico: Coccophagus indefinitus Myartseva n. sp., a parasitoid of Parasaissetia nigra (Nietner) on mistletoe Phoradendron quadrangulare (Kunth) Griseb., huisache Acacia farnesiana (L.) Willd., and willow Salix humboldtiana Willd.; and Coccophagus tamaulipecus Myartseva n. sp. on an unidentified species of Coccidae on an unidentified plant. This represents a new host plant record and new parasitoid association for Parasaissetia nigra on mistletoe.

Key words: Aphelinidae, Coccophagus, new species, mistletoe, Tamaulipas, Mexico.

\section{INTRODUCCIÓN}

El género Coccophagus Westwood pertenece a la familia de avispas parasitoides Aphelinidae de la superfamilia Chalcidoidea. Este género incluye más de 250 espe-

Recibido: 04/06/2013; aceptado: 03/12/2013. 
cies descritas a nivel mundial y presenta distribución cosmopolita (Noyes 2012). Las especies de Coccophagus son endoparasitoides primarios de Coccoidea, principalmente de escamas suaves (familia Coccidae). Los machos (ecto o endoparasitoides) generalmente son hiperparasitoides, incluyendo las hembras de su propia especie. De México se conocen 27 especies de Coccophagus, incluyendo 17 especies nuevas descritas durante las investigaciones de los Aphelinidae de México efectuadas por los autores (Myartseva et al. 2012). La primera clave para la identificación de 23 especies mexicanas de Coccophagus fue publicada en 2006 (Myartseva 2006). En varias de las publicaciones de los autores se presentan datos sobre hospederos y la importancia económica de especies de Coccophagus (Myartseva et al. 2004, 2010a, 2010b; Myartseva \& Ruiz-Cancino 2011; Ruiz-Cancino et al. 2006). Según Compere (1931), Annecke \& Insley (1974) y Hayat (1998), en Coccophagus se reconocen siete grupos de especies: malthusi, lycimnia, tschirchii, pseudococci, varius, ochraceus y zebratus; en México se presentan especies de cuatro grupos: lycimnia, malthusi, ochraceus y varius.

Parasaissetia nigra es una escama de la familia Coccidae (Hemiptera) que ataca muchas plantas distintas a nivel mundial. En California, Estados Unidos, anteriormente fue una plaga importante ocasional en chirimoya, guayaba, mango y papaya pero ahora no es una plaga de importancia económica, aparentemente debido a las temperaturas extremas, baja humedad, parasitoides y enfermedades. En California y Florida presenta una generación completa y una parcial por año (Gill 1988).

Por su parte, el muérdago (Familia Loranthaceae) es una planta hemiparásita que ataca diversas especies de árboles y arbustos en México, mermando la producción de conos y semillas, y el crecimiento de los árboles en diámetro, altura y volumen (Vázquez et al. 2006).

El objetivo de este artículo fue describir dos especies nuevas de Coccophagus: $C$. indefinitus Myartseva sp. $\mathrm{n}$. del grupo lycimnia y C. tamaulipecus Myartseva sp. $\mathrm{n}$. del grupo malthusi obtenidas de cóccidos en Tamaulipas, México.

\section{MATERIAL Y MÉTODOS}

Se colectaron muestras de plantas infestadas con escamas suaves en Cd. Victoria, Tamaulipas, a las orillas del Río San Marcos, en muérdago Phoradendron quadrangulare (Kunth) Griseb. (Familia Loranthaceae) sobre huizache Acacia farnesiana (L.) Willd. (Familia Fabaceae) y sauce Salix humboldtiana Willd. (Familia Salicaceae). En Jaumave, Tamaulipas, a las orillas del Río Nogales, se obtuvieron muestras en muérdago Psyttacanthus sp. (Familia Loranthaceae) sobre mezquite Prosopis tamaulipana Burkart (Familia Fabaceae) y en un arbusto no identificado; para la toma de muestras se siguió el método propuesto por Noyes (1982). El material fue llevado al Museo de Insectos MIFA de la Facultad de Ingeniería y Ciencias de la Universidad Autónoma de Tamaulipas, donde se obtuvo la emergencia de parasitoides, los cuales 
fueron preservados en alcohol al 75\%. Posteriormente se prepararon laminillas en bálsamo de Canadá. Para estudiar los detalles de su morfología se utilizó un microscopio compuesto Leica CME mientras que para la identificación de las especies de Coccophagus se utilizaron varias claves (Compere 1931, Annecke \& Insley 1974, Hayat 1998, Myartseva 2006). Además, se estudiaron las descripciones originales de algunas especies de estos autores para comparar con las especies nuevas.

\section{RESULTADOS}

Se obtuvieron parasitoides solamente de la escama suave Parasaissetia nigra en muérdago Phoradendron quadrangulare en ramas de huizaches y en el arbusto no identificado. Dos especies nuevas de Coccophagus emergieron de dos especies de Coccidae. A continuación se describen ambas especies.

\section{Coccophagus indefinitus Myartseva, sp. nov.}

(Figs. 1-5)

Material. Holotipo hembra: México, Tamaulipas, Ciudad Victoria, Río San Marcos, ex Parasaissetia nigra (Nietner) en Phoradendron quadrangulare en ramas de huizaches Acacia farnesiana, 21.II.2013 (cols. E. Ruiz C., N. Y. Martínez R., B. Villarreal A., N. Rivera T.). Paratipos: misma localidad y planta hospedera, además en sauces Salix humboldtiana, 2 9 , 2.XI.2012 (cols. E. Ruiz C. y J. M. Coronado B.); Jaumave, Río Nogales, ex Coccidae en arbusto, 3 + , 12.X.2012 (col. S. N. Myartseva). Todos los materiales en laminillas. Otros materiales en alcohol: 14 $9,12 . X .2012$, 4ㅇ, 2.XI.2012, 1q, 21.II.2013, 3 + , 7.III.2013 (cols. E. Ruiz C., N. Y. Martínez R., B. Villarreal A., N. Rivera T., A. M. Castillo M., L. A. Camacho H., J. M. Coronado B. y P. M. Castillo F.). El holotipo y 3 paratipos están depositados en el Entomological Research Museum de la Universidad de California, Riverside, Estados Unidos; 2 paratipos y los materiales en alcohol en el Museo de Insectos de la Facultad de Ingeniería y Ciencias de la Universidad Autónoma de Tamaulipas, en Cd. Victoria, Tamaulipas, México.

Longitud del cuerpo $-0.8-1.0 \mathrm{~mm}$.

Hembra

Coloración. Cabeza negra con líneas amarillas vértice-occipitales, ocelares y oculares. Antena amarilla. Palpos maxilares ahumados. Mesoescudo negro. Escutelo amarillo con la base negruzca al nivel del primer par de setas escutelares. Metanoto amarillo, ahumado lateralmente. Ala anterior ligeramente ahumada, setas parduzcas. Todas las coxas negras, coxa posterior blancuzca en el ápice. El fémur anterior y medio son negruzcos con el 1/4-1/3 apical amarillo blancuzco, fémur posterior negro con el ápice amarillo blancuzco. Tibia anterior ligeramente ahumada, tibia media 


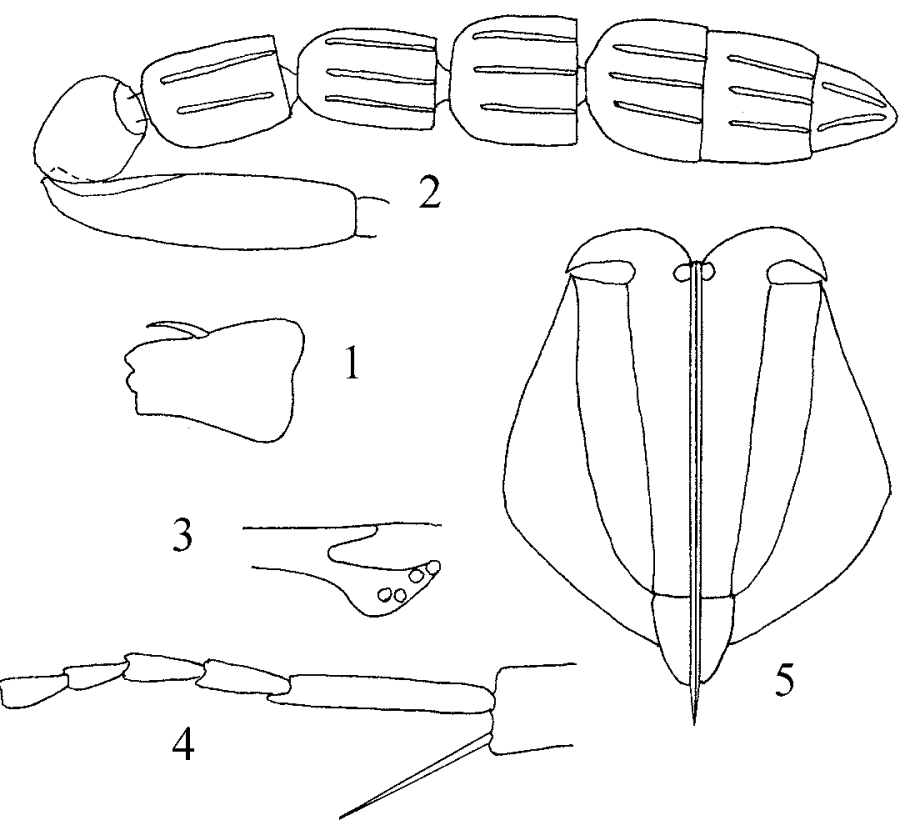

Figuras 1-5. Coccophagus indefinitus sp. nov., hembra: 1 - mandíbula, 2 - antena, 3 - vena estigmal, 4- tarso medio y espuela de la tibia media, 5 - ovipositor.

amarilla, tibia posterior amarilla, a veces con una pequeña área ahumada en la base dorsalmente. Tarso blancuzco, su segmento apical ahumado. Gáster negro, terguito 7 amarillento en el ápice, válvula 3 ligeramente parduzca.

Estructura. Cabeza 1.3 veces tan ancha como su altura. Frontovértice cerca de 0.6 veces tan ancho como la anchura de la cabeza. Distancia entre los ocelos posteriores ligeramente mayor que la distancia del ocelo al ojo. Ojos setosos y 2.1 veces tan largos como las mejillas. Mandíbula (Fig. 1) con dos dientes y una parte truncada. Antenas (Fig. 2) insertadas inmediatamente al nivel del margen inferior de los ojos. Distancia entre tórulos 0.6 veces tan larga como la distancia del tórulo al ojo. Radícula antenal 1.3 veces tan larga como ancha. Escapo 4.4 veces tan largo como ancho. Pedicelo 1.2 veces tan largo como ancho. Primer segmento funicular 1.4 veces tan largo como el pedicelo y 1.5 veces tan largo como ancho. Segundo segmento 0.8 veces tan largo como el primer segmento y 1.3 veces tan largo como ancho. Tercer segmento ligeramente más corto y tan largo como ancho. Maza antenal ligeramente más ancha que el funículo y tan larga como el escapo; los dos primeros segmentos de la maza tan anchos como largos. Segmentos flagelares 1 y 2 con dos sensilas longitudinales cada uno, los otros segmentos con 3 sensilas cada uno. Lóbulo medio del mesoescudo con muchas setas, axila con 2 setas largas, lóbulo lateral con 3 setas. Escutelo con 3 pares 
de setas largas, el tercer par es el más largo. Ala anterior densamente setosa, con una pequeña área sin pelos en la parte posterior y cerca de 2.4 veces tan larga como la anchura alar máxima. Vena marginal 1.4 veces tan larga como la vena submarginal, uncus de la vena estigmal elongado (Fig. 3), vena postmarginal muy corta. Fleco marginal del ala anterior muy corto, 0.1 veces tan largo como la anchura alar. Ala posterior cerca de 5 veces tan larga como la anchura alar máxima, su fleco marginal cerca de 0.4 veces tan largo como la anchura alar. Parte basal de la vena marginal con setas salientes. Fémur frontal y medio con una seta larga cerca del ápice, fémur posterior con unas pocas setas cortas cerca del ápice. Espuela de la tibia media (Fig. 4) 0.8 veces tan larga como el basitarso, basitarso tan largo como los tres segmentos tarsales siguientes juntos. Ovipositor (Fig. 5) no expuesto, 1.1 veces tan largo como la tibia media, tercera válvula cerca de 0.2 veces tan larga como el segundo valvífer y 0.7 veces tan larga como la espuela de la tibia media.

Macho desconocido.

Etimología. Del latín indefinites - incierto, no claro; en referencia a la posición sistemática de la especie.

Comentarios. Coccophagus indefinitus sp. n., pertenece al grupo de especies lycimnia y es cercana morfológicamente a otras dos especies, $C$. cowperi Girault y $C$. lycimnia (Walker). Se comparó la especie nueva con las descripciones y figuras de C. cowperi en Smith y Compere (1928), Annecke \& Insley (1974) y Hayat (1998). C. indefinitus n. sp., se distingue de C. cowperi en las siguientes características: 0.80 distal del escutelo es amarillo; coxa y fémur posterior con el ápice de amarillo a blanco; escapo amarillo como el flagelo; ala anterior 2.4 veces tan larga como ancha; fleco marginal del ala posterior 0.4 veces tan largo como la anchura alar; primer segmento funicular 1.5 veces tan largo como ancho, tercer segmento cuadrado; basitarso de la pata media tan largo como los tres siguientes segmentos tarsales juntos. En $C$. cowperi: 0.66 distal del escutelo es amarillo; coxa y fémur posterior negro; escapo negruzco; ala anterior 2.25 veces tan larga como ancha; fleco marginal del ala posterior 0.25 veces tan largo como la anchura alar; primer segmento funicular 2 veces tan largo como ancho, tercer segmento 0.75 veces tan largo como ancho; basitarso de la pata media tan largo como los cuatro siguientes segmentos tarsales juntos.

De C. lycimnia, la nueva especie difiere en lo siguiente: escapo, pedicelo y flagelo antenales amarillos; tibia posterior amarilla, sólo con una pequeña área ahumada cerca de la base dorsalmente; ala anterior con una pequeña área sin pelos en la parte posterior. En C. lycimnia, escapo y pedicelo antenales ahumados; tibia posterior negruzca; ala anterior sin una pequeña área sin pelos en la parte posterior.

Todos los autores, incluyendo Nikolskaya \& Yasnosh (1966), indicaron que $C$. cowperi y $C$. lycimnia son especies variables y muy cercanas. Por su parte, en los especímenes de la nueva especie no se encontró variabilidad en sus características.

Esta especie es cercana a C. tibialis Compere, por la coloración del cuerpo, escapo 
Myartseva et al.: Nuevas especies de Coccophagus en Tamaulipas

y patas que también pertenece al grupo lycimnia.

\section{Coccophagus tamaulipecus Myartseva, sp. n.}

(Figs. 6-10)

Material. Holotipo hembra: México, Tamaulipas, Jaumave, Río Nogales, ex Coccidae en arbusto no identificado, 12.X.2012 (col. S.N. Myartseva). El holotipo está depositado en el Entomological Research Museum de la Universidad de California, Riverside, Estados Unidos.

Longitud del cuerpo - $1.7 \mathrm{~mm}$.

Hembra

Coloración. Cabeza negra, antenas pardas, palpos labiales y maxilar parduzcos, segmento apical de los palpos maxilares negruzco. Cuerpo negro. Ala anterior muy ligeramente ahumada, setas parduzcas. Patas negras, tibia y tarso anteriores parduzcos, base del fémur medio con un anillo blancuzco, tarsos medios y posteriores blancuzcos, segmento apical negro parduzco, segmento preapical parduzco.

Estructura. Cabeza 1.2 veces tan ancha como alta. Frontovértice cerca de 0.4 veces tan ancho como la anchura de la cabeza. Ojos setosos, cerca de 1.8 veces tan largos como las mejillas. Mandíbula (Fig. 6) con dos dientes obtusos y una parte truncada. Frontovértice y cara con setas cortas. Antenas (Fig. 7) insertadas debajo del nivel del margen inferior de los ojos. Radícula antenal 1.4 veces tan larga como ancha. Escapo 4.4 veces tan largo como ancho. Pedicelo 1.5 veces tan largo como ancho. El primer segmento funicular es el más largo, 2.3 veces tan largo como ancho y 1.8 veces tan largo como el pedicelo. Segundo segmento 1.6 veces tan largo como ancho y cerca de 0.8 veces tan largo como el primer segmento. Tercer segmento ligeramente más corto y 1.2 veces tan largo como ancho. Primeros dos segmentos de la maza antenal más anchos que largos, tercer segmento 1.3 veces tan largo como ancho; maza antenal 1.4 veces tan larga como el primer segmento funicular y ligeramente más larga que los dos segmentos funiculares precedentes juntos. Todos los segmentos funiculares con 7 sensilas longitudinales en dos hileras, segmentos de la maza con 6 sensilas en una hilera. Lóbulo medio del mesoescudo con muchas setas dispersas y con 4 setas largas en cada lado. Axila con dos setas, lóbulo lateral con cuatro setas. Escutelo densamente setoso y con dos pares de setas largas en el ápice. Escultura del mesoescudo y escutelo consiste de una red de celdas grandes, más bien uniformes, rodeada de bordes esculpidos. Parte lateral de la axila con hileras de celdas situadas longitudinalmente. Propodeo dividido en la parte media. Ala anterior densamente setosa y 2.1 veces tan larga como la anchura alar máxima. Fleco marginal muy corto $(0.04 \mathrm{x})$. Vena submarginal con 9 setas largas a lo largo del margen anterior. Vena marginal ligeramente más larga que la vena submarginal. Vena estigmal (Fig. 8) con uncus corto y sésil, 


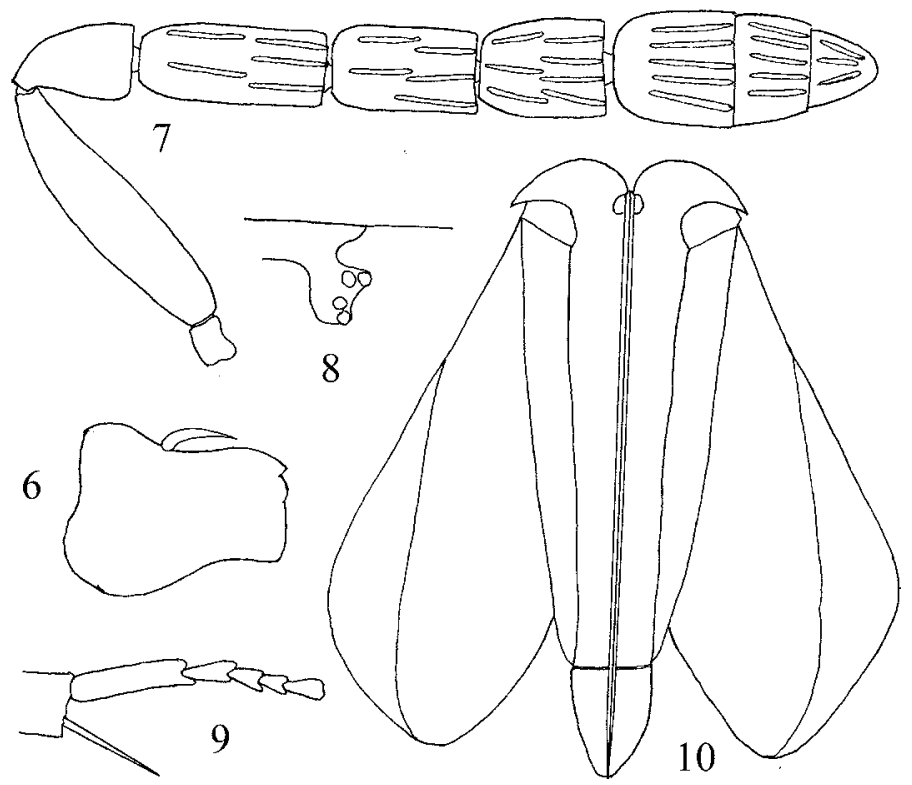

Figuras 6-10. Coccophagus tamaulipecus sp. nov., hembra: 6 - mandíbula, 7 - antena, 8 - vena estigmal, 9 - tarso medio y espuela de la tibia media, 10 - ovipositor.

vena postmarginal muy corta. Ala posterior 2.7 veces tan larga como la anchura alar máxima, su fleco marginal 0.1 veces tan largo como la anchura alar. Espuela de la tibia media (Fig. 9) peluda, 0.8 veces tan larga como el basitarso; segundo segmento tarsal 1.5 veces tan largo como el tercer segmento y tan largo como los dos segmentos tarsales siguientes juntos. Tibia posterior con una hilera dorsal de setas cortas y robustas. Ovipositor (Fig. 10) no expuesto, 1.1 veces tan largo como la tibia media; tercera válvula 0.2 veces tan larga como el segundo valvífer y 0.7 veces tan larga como la espuela de la tibia media.

Macho desconocido.

Etimología. El nombre de la especie es derivado de Tamaulipas, la entidad federativa mexicana donde se colectó el holotipo.

Comentarios. Coccophagus tamaulipecus sp. n., es cercano a C. quaestor Girault y a $C$. mexicensis Girault. Ambas especies fueron redescritas por Compere (1931) a partir de especímenes de México. La nueva especie difiere de C. quaestor en los siguientes caracteres: fémures medios de pardo oscuro a negro, con un anillo blancuzco en la base; tibia anterior de parduzca a negra, tarso parduzco; tibia media de parduzca a negra; pedicelo 1.5 veces tan largo como ancho; primer segmento funicular 2.3 veces tan largo como ancho; vena submarginal con 9 setas largas a lo largo del margen anterior; axila con dos setas más fuertes que las del mesoescudo; basitarso de la pata 
media ligeramente más corto que los siguientes tres segmentos tarsales juntos. En $C$. quaestor: fémures medios con ápice claro; tibia y tarso anteriores claros; tibia media ligeramente clara en el ápice; pedicelo más o menos tan ancho como largo; primer segmento funicular menos de 2 veces tan largo como ancho; vena submarginal con 11 setas a lo largo del margen anterior; axila con dos setas tan largas como las del mesoescudo; basitarso de la pata media tan largo como los siguientes 4 segmentos juntos.

De $C$. mexicensis, la nueva especie difiere por lo siguiente: tibias medias y posteriores negro parduzcas; pedicelo 1.5 veces tan largo como ancho; primer segmento funicular 2.3 veces tan largo como ancho; el primer segmento de la maza antenal es el más largo, tercer segmento más corto que el segundo. En C. mexicensis: tibias medias y posteriores de amarillo claro a blancuzco; pedicelo no mucho más largo que ancho; primer segmento funicular menos de 2 veces tan largo como ancho; segmentos de la maza antenal subiguales en longitud.

De acuerdo con Compere (1931), Annecke \& Insley (1974) y Hayat (1993), la nueva especie puede ser incluida en el grupo de especies malthusi, el cual incluye las especies de Coccophagus que tienen el escutelo densamente setoso además de los dos pares de setas más largas, un par apical visiblemente más largo y un par subapical relativamente más corto.

En este trabajo se reporta la escama Parasaissetia nigra como nuevo registro de asociación con muérdagos y a los afelínidos como nuevos registros como parasitoides de $P$. nigra en los muérdagos.

AgRAdecimientos. Al Dr. I. A. Gavrilov-Zimin (Zoological Institute, Russian Academy of Sciences) por la identificación del cóccido y a los Dres. Jacinto Treviño Carreón y Arturo Mora Olivo (Universidad Autónoma de Tamaulipas) por la identificación de los muérdagos. A las estudiantes de la Facultad de Ingeniería y Ciencias Nabil Yessenia Martínez Ruiz, Brenda Villarreal Alanís, Naylea Rivera Tovías, Adriana Michelle Castillo Martínez, Lizbeth Amalia Camacho Hernández y Patricia Marissa Castillo Flores, por su colaboración en las colectas de insectos en los muérdagos de Cd. Victoria. Al proyecto FIC-UAT "Muérdagos de Cd. Victoria, Tamaulipas" y al proyecto PROMEP "Taxonomía y ecología de fauna y micobiota en comunidades forestales y cultivos de México", de la Red de Cuerpos Académicos y a la Universidad Autónoma de Tamaulipas, por su apoyo para la realización de este trabajo.

\section{LITERATURA CITADA}

Annecke, D. P. \& Insley, H. P. 1974. The species of Coccophagus Westwood, 1833 from the Ethiopian region (Hymenoptera: Aphelinidae). Entomology Memoir, Department of Agricultural Technical Services, Republic of South Africa, 37: 1-62.

Compere, H. 1931. A revision of the species of Coccophagus, a genus of hymenopterous, coccid-inhabiting parasites. Proceedings of the U.S. Natural Museum, 78: 1-132.

Gill, R. J. 1988. The scale insects of California. Part 1. The soft scales (Homoptera: Coccidae). California Department of Food and Agriculture, Sacramento, $132 \mathrm{p}$.

Hayat, M. 1993. The malthusi-group of Coccophagus (Hymenoptera: Aphelinidae), with descriptions of three new species from India. Oriental Insects, 27: 175-184. 
Hayat, M. 1998. Aphelinidae of India (Hymenoptera: Chalcidoidea): a taxonomic revision. Memoirs on Entomology, International. Associated Publishers, Gainesville, Florida, U.S.A. 13: 1-416.

Myartseva, S. N. 2006. Review of Mexican species of Coccophagus Westwood, with a key and description of new species (Hymenoptera: Chalcidoidea: Aphelinidae). Zoosystematica Rossica, 15: 113-130.

Myartseva, S. N. \& Ruiz-Cancino, E. 2011. Parasitoides (Hymenoptera: Chalcidoidea) de Coccus (Hemiptera: Coccidae) asociados a Citrus en México. Dugesiana, 18: 65-72.

Myartseva, S. N., Ruiz-Cancino, E. \& Coronado-Blanco, J. M. 2004. Parasitoids (Hymenoptera: Chalcidoidea) of Saissetia spp. (Homoptera: Coccidae) in Mexico. Fruits, 59: 141-150.

Myartseva, S. N., Ruiz-Cancino, E. \& Coronado-Blanco, J. M. 2010a. Enemigos naturales (Chalcidoidea: Aphelinidae, Encyrtidae) de escamas suaves (Hemiptera: Coccidae) en México. Entomología Mexicana, 9: 967-970.

Myartseva, S. N., Ruiz-Cancino, E. \& Coronado-Blanco, J. M. 2010b. Parasitoides (Hymenoptera: Aphelinidae, Encyrtidae) de Differococcus argentinus (Morrison, 1919) (Hemiptera: Coccidae) en Tamaulipas, México. Dugesiana, 17: 125-128.

Myartseva, S. N., Ruiz-Cancino, E. \& Coronado-Blanco, J. M. 2012. Aphelinidae (Hymenoptera: Chalcidoidea) de importancia agrícola en México. Revisión y claves. Serie Avispas Parasíticas de Plagas y otros Insectos No. 8. Universidad Autónoma de Tamaulipas. México. 413 p. CD.

Nikolskaya, M. N. \& Yasnosh, V. A. 1966. Aphelinids of the European part of the USSR and the Caucasus (Hymenoptera, Aphelinidae). Opredeliteli po faune SSSR, 91. Moscow \& Leningrad, Nauka. 296 p. (in Russian).

Noyes, J. S. 1982. Collecting and preserving chalcid wasps (Hymenoptera: Chalcidoidea). Journal of Natural History, 16: 315-334.

Noyes, J. S. 2012. Universal Chalcidoidea Database [online]. Worldwide Web electronic publication. www.nhm.ac.uk/entomology/chalcidoids/index.html. (Last updated: June 2012).

Ruiz, C. E., Coronado, J. M. \& Myartseva, S. N. 2006. Situación actual del manejo de las plagas de los cítricos en Tamaulipas, México. Manejo Integrado de Plagas y Agroecología, 78: 94-100.

Smith, H. S. \& Compere, H. 1928. A preliminary report on the insect parasites of the black scale, Saissetia oleae (Bernard). University of California Publications in Entomology, 4: 231-334.

Vázquez, C. I., Villa R. A. \& Madrigal H. S. 2006. Los muérdagos (Loranthaceae) en Michoacán. INIFAP Libro Técnico No. 2, CIRPC Michoacán. México, 93 p. 